\title{
PRESCRIBED SINGULARITIES WITH WEIGHTS
}

\author{
I. A. Molnar
}

\begin{abstract}
We find the minimal weighted energy $\int_{\Omega} a(x)|\mathrm{D} u|^{n}$ of maps $u: \Omega \subset \mathbb{R}^{m+n} \rightarrow \mathbb{S}^{n}$ with prescribed singularities, where $a(\cdot)$ is a continuous positive weight. Our result extends previous ones of H. Brezis, J-M. Coron and E.H. Lieb (1986), G. Alberti, S. Baldo and G. Orlandi (2003), and V. Millot (2005).
\end{abstract}

\section{INTRODUCTION}

The problem of determining the minimum energy of a map $u$ with values in the unit sphere and with prescribed singularities was first investigated by H. Brezis, J-M. Coron, and E.H. Lieb in Harmonic Maps with Defects ([BCL86]), and it can be seen as a starting point in the analysis of some problems with applications in physics, like the ones concerning liquid crystals. The two-point problem was the following: given two points $A_{1}, A_{2}$ in $\Omega$ and $d \in \mathbb{Z}_{+}$,

$$
\begin{array}{r}
\operatorname{minimize} E_{A_{i}, d_{i}}(u):=\int_{\Omega}|\mathrm{D} u(x)|^{2} \mathrm{~d} x, \quad \text { when } u \in \mathrm{C}\left(\Omega \backslash\left\{A_{1}, A_{2}\right\} \subset \mathbb{R}^{3} ; \mathbb{S}^{2}\right), \\
\text { and } \operatorname{deg}\left(u, A_{1}\right)=-\operatorname{deg}\left(u, A_{2}\right)=d,
\end{array}
$$

where $\operatorname{deg}\left(u, A_{i}\right)$ is the usual topological degree of the restriction of $u$ to a small sphere $S \subset \Omega$ surrounding the point $A_{i}$ (and is independent of the specific choice of $S$ ). The answer to the problem was given as

$$
\inf _{u} E_{A_{i}, d_{i}}(u)=8 \pi d \operatorname{dist}\left(A_{1}, A_{2}\right),
$$

with the infimum not being, in general, achieved.

In this paper, we will treat a minimal energy problem that arises from a sequence of generalizations of the one above. Several variations were already proposed in [BCL86], and the following three are of interest to us. The first one was to consider more than two singularities (still a finite number of them), which lead to the concept of minimal connection between singularities with assigned degree. It was shown ([BCL86, Theorem 1.1]) that the solution of the problem corresponding to the points $A_{1}, \ldots, A_{N}$ and the degrees $d_{1}, \ldots, d_{N} \in \mathbb{Z}$ that satisfy $\sum_{i} d_{i}=0$, is

$$
\inf _{u} E_{A_{i}, d_{i}}(u)=8 \pi L, \quad \text { where } L \text { is the length of a minimal connection. }
$$

The key step in proving the inequality " $\leqslant$ " is the dipole construction, that is, the construction of an almost minimizer concentrated around each line segment associated to a minimal connection.

A second generalization consisted in placing the problem in a higher dimension, taking $u \in \mathrm{C}\left(\Omega \backslash\left\{A_{1}, \ldots, A_{N}\right\} \subset \mathbb{R}^{p} ; \mathbb{S}^{p-1}\right)$, and it was proved that the least energy is given in this case by

$$
\inf _{u} \int_{\mathbb{R}^{p}}|\mathrm{D} u(x)|^{p-1} \mathrm{~d} x=\sigma_{p}(p-1)^{(p-1) / 2} L,
$$


where $\sigma_{p}$ represents the $(p-1)$-dimensional measure of the sphere $\mathbb{S}^{p-1} \subset \mathbb{R}^{p}$. The third extension of the two-point problem, relevant here, was to consider a situation where the energy has the homogeneity of an area ([BCL86, section VIII.C]), for example minimize

$$
E_{\Gamma}(u):=\int_{\mathbb{R}^{3}}|\mathrm{D} u(x)| \mathrm{d} x, \quad \text { for } \quad u \in \mathrm{C}\left(\Omega \backslash \Gamma \subset \mathbb{R}^{3} ; \mathbb{S}^{1}\right) \text { with } \operatorname{deg}(u, \Gamma)=d,
$$

where $\Gamma$ is a given rectifiable, oriented Jordan curve in $\mathbb{R}^{3}$, and $d \in \mathbb{Z}$ is fixed. In the special case where the curve is planar, it was proved that

$$
\inf _{u} E_{\Gamma}(u)=2 \pi|d| \inf \left\{\operatorname{area}(\mathcal{S}) ; \mathcal{S} \text { surface in } \mathbb{R}^{3}, \partial \mathcal{S}=\Gamma\right\} .
$$

Moreover, the authors raise the same question in arbitrary dimension and codimension, more specifically

$$
\begin{aligned}
& \operatorname{minimize} E_{M_{0}}(u):=\int_{\Omega}|\mathrm{D} u(x)|^{n} \mathrm{~d} x, \\
& \text { for } u \in \mathrm{C}\left(\Omega \backslash M_{0} \subset \mathbb{R}^{m+n} ; \mathbb{S}^{n}\right), \text { and } \operatorname{deg}\left(u, M_{0}\right)=d \text {, }
\end{aligned}
$$

where $M_{0}$ is an $(m-1)$-dimensional boundaryless manifold in $\mathbb{R}^{m+n}$, and $d \in \mathbb{Z}$. Here, $\operatorname{deg}\left(u, M_{0}\right)$ represents the degree of the restriction of $u$ to a $n$-dimensional sphere $S$ that links with $M_{0}$ (i.e., $S$ is the boundary of a well-oriented $(n+1)$-dimensional disk that transversally intersects $M$ in a single point), and does not depend on the choice of $S$. They suggest that the solution should have the same form as (3), but the formula was afterwards rectified by F. Almgren, W. Browder, and E.H. Lieb in the paper Co-Area, Liquid Crystals, and Minimal Surfaces ([ABL88]).

In [ABL88], the authors first provide a new proof of the inequality " $\geqslant$ " in (1), which uses the coarea formula and current slicing, and which can more easily be extended to higher dimensions. In the main theorem, they give the solution of the problem (4), put in the context of integral currents, and then give an outline of the proof. The result obtained was, roughly, that

$$
\inf _{u} E_{M_{0}}(u)=n^{n / 2} \sigma_{n+1} \inf \left\{\operatorname{mass}(T) ; T \text { is an integral current with } \partial T=d M_{0}\right\} .
$$

A similar setting for this problem is given in the article of G. Alberti, S. Baldo and G. Orlandi, entitled Functions with prescribed singularities ([ABO03]). Their main interest was to study the image of the distributional Jacobian Ju, which is known to describe in some sense the topological singularities of the map $u$. They have shown an identification between $\star \mathrm{J} u$, the current associated, via the Hodge-type operator $\star$, to the distributional Jacobian of a $\mathbb{S}^{n}$-valued map, and the boundary of a rectifiable current of codimension $n$. More precisely (see [ABO03, Theorem 3.8 and Theorem 5.6]), they have proved, on one side, that given $u \in \mathrm{W}^{1, n}\left(\Omega, \mathbb{S}^{n}\right)$, there exists a rectifiable current $T$ that satisfies the inequality

$$
\sigma_{n+1} \times \operatorname{mass}(T) \leqslant \int_{\Omega}|\mathrm{D} u(x)|^{n} \mathrm{~d} x,
$$

and the condition $\star \mathrm{J} u=\frac{\sigma_{n+1}}{n+1} \partial T$, and, conversely, that for a given current $T$, there exists a map $u$ that verifies the above condition on its distributional Jacobian, and such that

$$
\int_{\Omega}|\mathrm{D} u(x)|^{n} \mathrm{~d} x \leqslant c(m, n) \times \operatorname{mass}(T),
$$

where $c(m, n)$ is a constant that depends only on $m$ and $n$.

Their approach was the following: for the proof of the upper bound, they used a dipole construction together with a result concerning the approximation of integral currents by 
polyhedral ones, and a rather elaborate induction argument. The lower bound of the energy was obtained, in short, in the same manner as in [ABL88], through the use of the coarea formula. They presented the proof in much more detail though, giving a variant of the coarea formula that involves the distributional Jacobian, or more generally - for a map taking values in a Riemannian manifold $M$ - the pullback of the volume form on $M$. This allows them to prove that the current $T$ in (5) can be taken to be the slice determined by $u$ at a point $y \in \mathbb{S}^{n}$ in the subset of $\Omega$ where $u$ is approximately differentiable.

In our paper, we will place the problem in the same setting proposed in [ABO03], but we discuss the associated weighted energy problem. We will also follow closely their strategy and see that, for the upper bound, replacing the dipole construction in [ABO03] with the one originally introduced in [BCL86], and being careful at the estimates obtained in the induction process, their method actually yields $c(m, n)=c(n)=n^{n / 2} \sigma_{n+1}$ in (6), which is exactly the constant from (2). Also, inequality (5) is still valid for this larger constant, instead of $\sigma_{n+1}$.

The problem of the weighted energy was studied, in the classical context, by V. Millot in Energy with weight for $\mathbb{S}^{2}$-valued maps with prescribed singularities ([Mil05]), where he considered the problem (1) with energy

$$
E_{A_{i}, d_{i}}(u, a):=\int_{\Omega} a(x)|\mathrm{D} u(x)|^{2} \mathrm{~d} x,
$$

for a measurable function $a(\cdot)$ that satisfies $0<\lambda \leqslant a(\cdot) \leqslant \Lambda$, for some given constants $\lambda$ and $\Lambda$. He showed that the formula (1) still holds, provided that the distance function used in the expression of the length of the connection is conveniently chosen. He adopted the following definition for the length of a segment $\left(A_{1}, A_{2}\right)$ in $\mathbb{R}^{3}$ :

$$
\ell_{a}\left(A_{1}, A_{2}\right)=\liminf _{\varepsilon \rightarrow 0} \frac{1}{\pi \varepsilon^{2}} \int_{\Xi\left(A_{1}, A_{2} ; \varepsilon\right)} a(x) \mathrm{d} x
$$

where $\Xi\left(A_{1}, A_{2} ; \varepsilon\right)$ denotes the set $\left\{x \in \Omega ;\left|x-x_{0}\right| \leqslant \varepsilon\right.$, for some $\left.x_{0} \in\left(A_{1}, A_{2}\right)\right\}$ obtained by thickening the segment $\left(A_{1}, A_{2}\right)$. The new distance was then defined by

$$
\operatorname{dist}_{a}(A, B)=\inf \sum_{k=1}^{N} \ell_{a}\left(A_{k}, A_{k+1}\right),
$$

where the infimum is taken over all the polygonal lines $\left(A_{1}, \ldots, A_{N+1}\right)$ connecting the points $A$ and $B$, such that dist ${ }_{1}$ is the usual Euclidean distance in $\mathbb{R}^{3}$.

We will consider the case where the function $a(\cdot)$ is continuous, but where, as in [ABO03], the dimension and codimension are arbitrary. The exact statement of the main result will be given at the end of the next section. As we already mentioned, the strategy used is the one from [ABO03]. However, in order to be able to take into consideration the fact that we are placed in a heterogeneous setting, we will have to define a new measure on $\Omega$, by analogy with the distance dist $_{a}$ defined in [Mil05]. Naturally, the area of $\mathcal{S}$ in (3) must be replaced with the integral $\int_{\mathcal{S}} a(x) \mathrm{d} \mathcal{H}^{2}(x)$, so we will define a modified Hausdorff measure $\mathcal{H}_{a}^{h}$ (which in fact makes sense even if $a(\cdot)$ is only $\mathcal{L}^{n}$-measurable), and which for $a \equiv 1$ becomes the usual $h$-dimensional Hausdorff measure on $\mathbb{R}^{n}$.

\section{Preliminaries and statement of the theorem}

In this section we present the background of the problem. We will consider $\Omega$ to be a bounded and smooth open subset of $\mathbb{R}^{p}$, and $M$ a smooth oriented $m$-dimensional submanifold of $\mathbb{R}^{n+1}$, with the dimensions satisfying $m \leqslant n+1 \leqslant p$. 
2.1. Rectifiable currents. We recall here the definitions and the basic properties of the currents with which we are concerned. For further details, see [Pap02, Chapter 24], [Mor09, Chapter 4], [GMS98, Chapter 2, Section 2], and, of course, [Fed69].

Basically, a $h$-dimensional current is a generalization of a distribution, where the place of the test functions $\mathrm{C}_{\mathrm{c}}^{\infty}(\Omega, \mathbb{R})$ is taken by the $h$-dimensional differential forms with compact support $\mathcal{D}^{h}(\Omega)$. More precisely, $\mathcal{D}_{h}(\Omega)$, the space of $h$-dimensional currents on $\Omega$, is the topological dual of $\mathcal{D}^{h}(\Omega)$ with respect to the topology induced by the family of seminorms $\nu_{K}^{i}(\eta)=\sup _{j \leqslant i, x \in K}\left\|\mathrm{D}^{j} \eta(x)\right\|$ on each $\mathcal{D}_{K}^{h}(\Omega):=\left\{\eta \in \mathcal{D}^{h}(\Omega) ; \operatorname{supp} \eta \subset K\right\}$. The vector space $\mathcal{D}_{h}(\Omega)$ is then endowed with the weak topology, that is, the pointwise convergence of currents $T_{i} \rightarrow T \Longleftrightarrow T_{i}(\eta) \rightarrow T(\eta), \forall \eta \in \mathcal{D}^{h}(\Omega)$. The mass of $T$ is defined by $M(T)=\sup _{\|\eta(x)\| \leqslant 1, \forall x}|T(\eta)|$.

The boundary of $T$ is the current $\partial T \in \mathcal{D}_{h-1}(\Omega)$ defined by $\partial T(\mu):=T(\mathrm{~d} \mu)$, for every $\mu \in \mathcal{D}^{h-1}(\Omega)$, in consistency with Stokes' theorem.

The class of rectifiable currents $\mathcal{R}_{h}(\Omega)$ consists of currents $T$ that can be expressed by an integral formula

$$
T(\eta)=\int_{\widetilde{T}} \theta_{T}(x)\left\langle\eta(x), \tau_{T}(x)\right\rangle \mathrm{d} \mathcal{H}^{h}(x), \quad \forall \eta \in \mathcal{D}^{h}(\Omega) .
$$

where $\widetilde{T}$ is a compact, $h$-rectifiable subset of $\Omega, \theta_{T}: \widetilde{T} \rightarrow \mathbb{Z}_{+}$is a $\mathcal{H}^{h}$-measurable function called the multiplicity of $T$, and $\tau_{T}$ is an orientation on $\widetilde{T}$. The mass of $T$ then becomes

$$
M(T)=\int_{\widetilde{T}} \theta_{T}(x) \mathrm{d} \mathcal{H}^{h}(x) .
$$

Given an $\mathcal{H}^{h}$-integrable function $a(\cdot): \Omega \rightarrow \mathbb{R}$, we can define another current $T\left\llcorner a \in \mathcal{R}^{h}(\Omega)\right.$, by

$$
T\left\llcorner a(\eta)=\int_{\widetilde{T}} a(x) \theta_{T}(x)\left\langle\eta(x), \tau_{T}(x)\right\rangle \mathrm{d} \mathcal{H}^{h}(x), \forall \eta \in \mathcal{D}^{h}(\Omega) .\right.
$$

If $a(\cdot)$ is the characteristic function of a $\mathcal{H}^{h}$-measurable set $A$, then $T\left\llcorner\chi_{A}\right.$ is also written as $T\llcorner A$, and is called the restriction of $T$ to $A$.

Given a $h$-rectifiable set $S \subset \Omega$ oriented by $\tau_{S}$, we let $\llbracket S \rrbracket$ denote the current associated to $S$ through the same expression as in (8), where the multiplicity is considered equal to 1 . If $S$ is a smooth oriented compact manifold, then, by Stokes' theorem, we have that $\partial \llbracket S \rrbracket=\llbracket \partial S \rrbracket$.

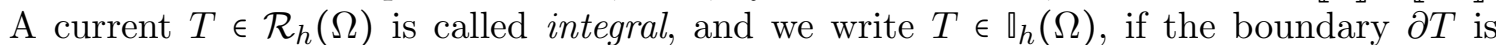
also rectifiable. By the Closure Theorem [Fed69, Theorem 4.2.16], a necessary and sufficient condition for a rectifiable current to be integral is that its boundary has finite mass.

The class of integral flat currents $\mathcal{F}_{h}(\Omega)$ is defined as the set of currents $T$ that can be written as the sum $P+\partial Q$, with $P \in \mathcal{R}_{h}(\Omega)$, and $Q \in \mathcal{R}_{h+1}(\Omega)$. The flat (semi-)norm on $\mathcal{F}_{h}(\Omega)$ is defined by

$$
\mathcal{F}(T):=\inf \left\{M(P)+M(Q) ; T=P+\partial Q, P \in \mathcal{R}_{h}(\Omega), Q \in \mathcal{R}_{h+1}(\Omega)\right\} .
$$

The space of integral polyhedral currents $\mathcal{P}_{h}(\Omega)$ consists of rectifiable currents that can be written as a finite sum of $h$-dimensional simplexes with constant integer multiplicities. It represents a dense subset of $\mathcal{F}_{h}(\Omega)$, with respect to the flat norm. The inclusion relations between the previous classes of currents are the following:

$$
\mathcal{P}_{h}(\Omega) \subset \mathbb{Q}_{h}(\Omega) \subset \mathcal{R}_{h}(\Omega) \subset \mathcal{F}_{h}(\Omega) \subset \mathcal{D}_{h}(\Omega) .
$$


2.2. The pullback $u^{\sharp} \omega$ of the volume form of an oriented manifold. Denote by $\omega$ the (standard) volume form on $M$, that is, the differential $m$-form on $M$ which associates to each point $y \in M$ the $m$-linear alternating map on $\mathrm{T}_{y} M$ that satisfies

$$
\omega(y)\left(\tau_{1}, \ldots, \tau_{m}\right)=1,
$$

for any $\left\{\tau_{1}, \ldots, \tau_{m}\right\}$ positively oriented orthonormal basis of $\mathrm{T}_{y} M$. Since the top-dimensional forms constitute a 1-dimensional vector space, we see that $\omega$ is necessarily given by

$$
\omega(y)\left(w_{1}, \ldots, w_{m}\right)=\operatorname{det}\left[w_{1}, \ldots, w_{m}, v_{1}, \ldots, v_{n-m+1}\right],
$$

for every $y \in M, w_{1}, \ldots, w_{m} \in \mathrm{T}_{y} M$, and any $\left\{v_{1}, \ldots, v_{n-m+1}\right\} \subset \mathbb{R}^{n+1}$ a positively oriented orthonormal basis of $\mathrm{N}_{y} M=\mathrm{T}_{y} M^{\perp}$. The value of the above determinant is independent of the choice of basis of the normal space since passing to another one leads to the multiplication of the matrix in the right-hand side by an orthogonal matrix of determinant equal to 1 .

Suppose $u: \Omega \rightarrow M$ is a differentiable map. We can then consider the pullback of the volume form $\omega$ with respect to $u$, which is the $m$-form on $\Omega$ defined by

$$
\left(u^{\sharp} \omega\right)(x)\left(v_{1}, \ldots, v_{m}\right)=\omega(u(x))\left(\mathrm{d} u_{x}\left(v_{1}\right), \ldots, \mathrm{d} u_{x}\left(v_{m}\right)\right),
$$

for every $x \in \Omega$ and $v_{1}, \ldots, v_{m} \in \mathbb{R}^{p}$. Remark that in [ABO03], the notation $\mathrm{J}_{\omega} u$ is used instead of $u^{\sharp} \omega$, to emphasise the role that it plays in the definition of the distributional Jacobian.

For every $x \in \Omega$, the form $\left(u^{\sharp} \omega\right)(x)$ is an element of the space $\Lambda^{m}\left(\mathbb{R}^{p}\right)$ of the $m$-covectors in $\mathbb{R}^{p}$, which can be endowed with the Euclidean norm, meaning that if we let $\mathrm{I}(m, p)$ denote the set of ordered multi-indices $\alpha=\left(\alpha_{1}, \ldots, \alpha_{m}\right)$ with $1 \leqslant \alpha_{1}<\cdots<\alpha_{m} \leqslant p$, and $\left\{\mathrm{d} x_{1}, \ldots, \mathrm{d} x_{p}\right\}$ is the dual base of an orthonormal basis $\left\{e_{1}, \ldots, e_{p}\right\}$ of $\mathbb{R}^{p}$, then

$$
\left(u^{\sharp} \omega\right)(x)=\sum_{\alpha \in \mathrm{I}(m, p)}\left(u^{\sharp} \omega\right)(x)\left(e_{\alpha_{1}}, \ldots, e_{\alpha_{m}}\right) \mathrm{d} x_{\alpha_{1}} \wedge \cdots \wedge \mathrm{d} x_{\alpha_{m}},
$$

and

$$
\left|\left(u^{\sharp} \omega\right)(x)\right|^{2}=\sum_{\alpha \in \mathrm{I}(m, p)}\left|\left(u^{\sharp} \omega\right)(x)\left(e_{\alpha_{1}}, \ldots, e_{\alpha_{m}}\right)\right|^{2} .
$$

With this, we can see that $\left|\left(u^{\sharp} \omega\right)(x)\right|$ is simply the Jacobian $\llbracket \mathrm{D} u(x) \rrbracket$ of the $m \times(n+1)$-matrix $\mathrm{D} u(x)$ of the differential of $u$ at $x$, that appears in the classical coarea formula, that is,

$$
\left|\left(u^{\sharp} \omega\right)(x)\right|=\llbracket \mathrm{D} u(x) \rrbracket=\left[\operatorname{det}\left(\mathrm{D} u(x) \mathrm{D} u(x)^{\star}\right)\right]^{1 / 2} .
$$

Indeed, writing the matrix of the differential of $u$ in $x$ with respect to the canonical basis $\left\{e_{1}, \ldots, e_{p}\right\}$ in $\mathbb{R}^{p}$ and an orthonormal basis $\left\{\tau_{1}, \ldots, \tau_{m}\right\}$ in $\mathrm{T}_{u(x)} M$,

$$
\mathrm{d} u_{x}\left(e_{i}\right)=\sum_{j=1}^{m} \lambda_{j i} \tau_{j}=\lambda_{i} \cdot\left(\tau_{1}, \ldots, \tau_{m}\right), \quad \forall i=1, \ldots, p,
$$

clearly gives us

$$
\left|\left(u^{\sharp} \omega\right)(x)\right|^{2}=\sum_{\alpha \in \mathrm{I}(m, p)}\left|\operatorname{det}\left[\lambda_{\alpha_{1}}, \ldots, \lambda_{\alpha_{m}}\right]\right|^{2}=\llbracket \mathrm{D} u(x) \rrbracket^{2} .
$$

Remark that the quantity $\left|\left(u^{\sharp} \omega\right)(x)\right|$ is called the $m$-dimensional Jacobian of $u$ at $x$ in [ABL88]. We will later make use of the following estimate:

$$
\left|\left(u^{\sharp} \omega\right)(x)\right| \leqslant m^{-m / 2} \times|\mathrm{D} u(x)|^{m}, \quad \text { for all } x \in \Omega,
$$

that can be found also in [ABL88, Appendix A.1.3]. In fact, it was obtained before that in [BCL86, (8.5)] in the case where $M \equiv \mathbb{S}^{m}$. In that context, we have $\left|\left(u^{\sharp} \omega\right)(x)\right|=|D|$, with $D$ 
representing the vector field $\left(D_{1}, \ldots, D_{p}\right)$ that is defined by $D_{j}=\operatorname{det}\left[\partial_{1} u, \ldots, \partial_{j-1} u, u, \partial_{j+1} u, \ldots, \partial_{p} u\right]$, $j=1, \ldots, p$. To verify inequality (10), suppose first that $u$ is a submersion at the point $x \in \Omega$, that is, the differential

$$
\mathrm{d} u_{x}: \mathbb{R}^{p} \rightarrow \mathrm{T}_{u(x)} M
$$

is surjective and so its null space has dimension $(p-m)$. If we choose $\left\{e_{1}, \ldots, e_{p}\right\}$ to be an orthonormal basis in $\mathbb{R}^{p}$ such that $\operatorname{ker}\left(\mathrm{d} u_{x}\right)=\operatorname{span}\left\{e_{m+1}, \ldots, e_{p}\right\}$, then only one term in the expression of $\left|\left(u^{\sharp} \omega\right)(x)\right|$ is non zero, namely the one indexed by $\alpha=(1, \ldots, m)$, hence

$$
\left|\left(u^{\sharp} \omega\right)(x)\right|=\left|\operatorname{det}\left(\mathrm{d} u_{x}\left(e_{1}\right), \ldots, \mathrm{d} u_{x}\left(e_{m}\right), v_{1}, \ldots, v_{n-m+1}\right)\right|,
$$

where $\left\{\nu_{1}, \ldots, \nu_{n-m+1}\right\}$ is an orthonormal basis in $\mathrm{N}_{u(x)} M$. By Hadamard's inequality and the arithmetic-geometric mean inequality we obtain

$$
\begin{aligned}
\left|\left(u^{\sharp} \omega\right)(x)\right|^{2} & \leqslant\left|\mathrm{~d} u_{x}\left(e_{1}\right)\right|^{2} \ldots\left|\mathrm{d} u_{x}\left(e_{m}\right)\right|^{2} \\
& \leqslant\left[\frac{1}{m}\left(\left|\mathrm{~d} u_{x}\left(e_{1}\right)\right|^{2}+\cdots+\left|\mathrm{d} u_{x}\left(e_{m}\right)\right|^{2}\right)\right]^{m}=\left(\frac{|\mathrm{D} u(x)|^{2}}{m}\right)^{m} .
\end{aligned}
$$

If however, $\mathrm{d} u_{x}$ is not surjective, then any $m$ vectors $\mathrm{d} u_{x}\left(e_{\alpha_{1}}\right), \ldots, \mathrm{d} u_{x}\left(e_{\alpha_{m}}\right)$ are linearly dependent, and so, $\left|\left(u^{\sharp} \omega\right)(x)\right|$ is zero.

Notice that everything remains true almost everywhere if we suppose $u: \Omega \rightarrow M$ is only Lipschitz. Also, the definition of the pullback of $\omega$ makes sense if $u$ is merely in $\mathrm{W}^{1, m}(\Omega, M)$, and then $\left|\left(u^{\sharp} \omega\right)(\cdot)\right|$ belongs to $\mathrm{L}^{1}(\Omega)$.

Rather than the form $u^{\sharp} \omega$, the associated current $\star u^{\sharp} \omega$ will be of more interest in what follows. The action of the Hodge-type operator $\star$ on $u^{\sharp} \omega$ is given by

$$
\star\left(u^{\sharp} \omega\right)(x)=\sum_{\alpha \in \mathrm{I}(p-m, p)} \operatorname{sgn}(\alpha, \bar{\alpha})\left(u^{\sharp} \omega\right)(x)\left(e_{\bar{\alpha}_{1}} \ldots, e_{\bar{\alpha}_{m}}\right) e_{\alpha_{1}} \wedge \cdots \wedge e_{\alpha_{p-m}},
$$

which thus becomes a $(p-m)$-current on $\Omega$. Here, $\bar{\beta}$ denotes the multi-index in $\mathrm{I}(m, p)$ that complements the element $\beta \in \mathrm{I}(p-m, p)$.

2.3. An integral representation of the current $\star u^{\sharp} \omega$ via the coarea formula. Suppose $u: \Omega \rightarrow M$ is a Lipschitz map. The coarea formula (see [Fed59, Theorem 3.1], [EG92, Section 3.4]) states that given an $\mathcal{L}^{p}$-integrable function $a(\cdot): \Omega \rightarrow \mathbb{R}$, and an $\mathcal{L}^{p}$-measurable set $A \subset \mathbb{R}^{p}$, we have that, for $\mathcal{H}^{m}$-almost every $y \in M$, the level set $u^{-1}(y)$ is $\mathcal{H}^{p-m}$-rectifiable, and

$$
\int_{A} a(x) \llbracket \mathrm{D} u(x) \rrbracket \mathrm{d} x=\int_{M}\left(\int_{u^{-1}(y) \cap A} a(x) \mathrm{d} \mathcal{H}^{p-m}(x)\right) \mathrm{d} \mathcal{H}^{m}(y),
$$

hence, also, for any continuous function $\rho: M \rightarrow \mathbb{R}$,

$$
\int_{A} \rho(u(x)) \llbracket \mathrm{D} u(x) \rrbracket \mathrm{d} x=\int_{M} \rho(y) \mathcal{H}^{p-m}\left(u^{-1}(y) \cap A\right) \mathrm{d} \mathcal{H}^{m}(y),
$$

assuming that the left-hand side of (12) is integrable.

If we choose the set $A$ conveniently, we see that for $\mathcal{H}^{p-m}$-almost every $y$ in $M$, the Jacobian $\llbracket \mathrm{D} u(x) \rrbracket=\left|u^{\sharp} \omega(x)\right|$ is defined and is non zero, for $\mathcal{H}^{p-m}$-almost any $x$ in $u^{-1}(y)$. At every such points $x$, the differential $\mathrm{d} u_{x}$ vanishes on the tangent space of the level set $u^{-1}(y)$, and so, using a basis $\left\{e_{1}, \ldots, e_{p}\right\}$ of $\mathbb{R}^{p}$ for which $\operatorname{Tan}_{x} u^{-1}(y)=\operatorname{span}\left\{e_{1}, \ldots, e_{p-m}\right\}$, we have

$$
\star u^{\sharp} \omega(x)=\omega(u(x))\left(\mathrm{d} u_{x}\left(e_{p-m+1}\right), \ldots, \mathrm{d} u_{x}\left(e_{p}\right)\right) e_{1} \wedge \cdots \wedge e_{p-m},
$$


hence, after normalisation, $\star u^{\sharp} \omega$ defines an orientation of the rectifiable set $u^{-1}(y)$. Thus, $u^{-1}(y)$ becomes a rectifiable current of multiplicity equal to 1 , that is,

$$
\llbracket u^{-1}(y) \rrbracket(\eta)=\int_{u^{-1}(y)}\left\langle\eta(x), \frac{\star u^{\sharp} \omega(x)}{\left|\star u^{\sharp} \omega(x)\right|}\right\rangle \mathrm{d} \mathcal{H}^{p-m}(x),
$$

for all $\eta \in \mathcal{D}^{p-m}(\Omega)$, and for $\mathcal{H}^{m}$-almost every $y \in M$. Equivalently, $\llbracket u^{-1}(y) \rrbracket$ is the $\mathbb{R}^{p-m}$-valued measure $\frac{\star u^{\sharp} \omega(x)}{\left|\star u^{\sharp} \omega(x)\right|} \mathcal{H}^{p-m}\left\llcorner\left\{u^{-1}(y)\right\}\right.$, so its total variation is $\left|\llbracket u^{-1}(y) \rrbracket\right|=\mathcal{H}^{p-m}\left\llcorner\left\{u^{-1}(y)\right\}\right.$, meaning that for all $\varphi \in \mathrm{C}_{\mathrm{c}}(\Omega)$, we have

$$
\left|\llbracket u^{-1}(y) \rrbracket\right|(\varphi)=\int_{u^{-1}(y)} \varphi(x) \mathrm{d} \mathcal{H}^{p-m}(x) .
$$

By approximating $\varphi$ with simple functions, we see that $\int_{u^{-1}(y)} \varphi(x) \mathrm{d} \mathcal{H}^{p-m}(x)$ is $\mathcal{H}^{m}$-measurable (as pointwise limit of $\mathcal{H}^{m}$-measurable functions), that is, the mapping $y \mapsto\left|\llbracket u^{-1}(y) \rrbracket\right|$ is weak$\star$-measurable. Applying the coarea formula and the monotone convergence theorem, we obtain that, for any $\varphi \in \mathrm{C}_{\mathrm{c}}(\Omega)$,

$$
\int_{\Omega}|\rho(u(x))| \cdot\left|u^{\sharp} \omega(x)\right| \varphi(x) \mathrm{d} x=\int_{M}|\rho(y)| \cdot\left|\llbracket u^{-1}(y) \rrbracket\right|(\varphi) \mathrm{d} \mathcal{H}^{m}(y) .
$$

The integrals are finite, because we have

$$
\begin{aligned}
& \int_{M}|\rho(y)| \cdot|| \llbracket u^{-1}(y) \rrbracket|(\varphi)| \mathrm{d} \mathcal{H}^{m}(y) \leqslant \sup _{\Omega}|\varphi| \int_{M}|\rho(y)| \mathcal{H}^{p-m}\left(u^{-1}(y)\right) \mathrm{d} \mathcal{H}^{m}(y) \\
& =\sup _{\Omega}|\varphi| \int_{\Omega}|\rho(u(x))| \cdot\left|u^{\sharp} \omega(x)\right| \mathrm{d} x \leqslant m^{-m / 2} \sup _{\Omega}|\varphi| \sup _{M}|\rho| \int_{\Omega}|\mathrm{D} u(x)|^{m} \mathrm{~d} x,
\end{aligned}
$$

which is finite, since $\Omega$ is bounded, and thus, the map $\left|\llbracket u^{-1}(\cdot) \rrbracket\right|$ is weak- $\star$ - integrable. The same can be said about the function $\left|u^{\sharp} \omega(\cdot)\right|$, as it belongs to $L^{1}(\Omega)$, and so, we can express the equality (13) in short as

$$
\left|u^{\sharp}(\rho \omega)\right|=\int_{M}^{\star}|\rho(y)| \cdot\left|\llbracket u^{-1}(y) \rrbracket\right| \mathrm{d} \mathcal{H}^{m}(y),
$$

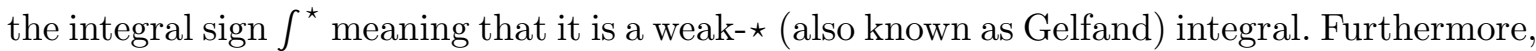
since the identity (13) is in fact true for every bounded function $\varphi \in \mathrm{L}^{1}(\Omega)$, we can take $\varphi$ as defined by the duality product

$$
\varphi(x)=\left\langle\star u^{\sharp}(\rho \omega)(x), \frac{\eta(x)}{\left|u^{\sharp}(\rho \omega)(x)\right|}\right\rangle,
$$

for any $\eta \in \mathcal{D}^{p-m}(\Omega)$, and we get

$$
\begin{aligned}
& \int_{\Omega}\left\langle\star u^{\sharp}(\rho \omega)(x), \eta(x)\right\rangle \mathrm{d} x \\
& \quad=\int_{M} \rho(y)\left[\int_{u^{-1}(y)}\left\langle\star u^{\sharp} \omega(x), \frac{\eta(x)}{\left|u^{\sharp} \omega(x)\right|}\right\rangle \mathrm{d} \mathcal{H}^{p-m}(x)\right] \mathrm{d} \mathcal{H}^{m}(y) .
\end{aligned}
$$

This can be also written as

$$
\star u^{\sharp}(\rho \omega)=\int_{M}^{\star} \rho(y) \llbracket u^{-1}(y) \rrbracket \mathrm{d} \mathcal{H}^{m}(y) .
$$

Suppose now that the map $u$ belongs to $\mathrm{W}^{1,1}(\Omega, M)$. In order to deduce an analogue of the coarea formula for Sobolev functions from the classical one, it is natural to try to use some 
Luzin type approximation results. We begin by covering $\Omega$, up to a Lebesgue null set $E \subset \Omega$, by a disjoint sequence $\Omega_{j}$ of measurable subsets in $\mathbb{R}^{p}$ with the property that the restriction $u_{j}$ of $u$ to every such set is Lipschitz (using for example [EG92, Theorem 3, Section 6.6.3]). We can then apply formula (11) to every $u_{j}: \Omega_{j} \rightarrow M$, to obtain

$$
\int_{A \cap \Omega_{j}} a(x)\left|u^{\sharp} \omega(x)\right| \mathrm{d} x=\int_{M}\left(\int_{u_{j}^{-1}(y) \cap \Omega_{j} \cap A} a(x) \mathrm{d} \mathcal{H}^{p-m}(x)\right) \mathrm{d} \mathcal{H}^{m}(y),
$$

for any $\mathcal{L}^{p}$-measurable set $A \subset \Omega$ and $\mathcal{L}^{p}$-integrable function $a(\cdot): \Omega \rightarrow \mathbb{R}$. Taking the sum over all $j$, we get,

$$
\int_{A} a(x)\left|u^{\sharp} \omega(x)\right| \mathrm{d} x=\int_{M}\left(\int_{u^{-1}(y) \backslash E \cap A} a(x) \mathrm{d} \mathcal{H}^{p-m}(x)\right) \mathrm{d} \mathcal{H}^{m}(y),
$$

at least when $a(\cdot)$ is nonnegative, where, in the left-hand, we used the fact that the set $E$ has Lebesgue measure zero. However, since the restriction $\left.u\right|_{E}$ is not Lipschitz, it need not have the Luzin N-property, hence $\mathcal{H}^{p-m}\left(u^{-1}(y) \cap A \cap E\right)$ is not necessarily equal to zero, so in the analogue of the coarea formula for $u$ we have to keep in mind that we have to remove the set $E$ from the usual level set $u^{-1}(y)$. What we can say about this set $E$ is that the function $u$ is almost everywhere approximately differentiable on its complement $\Omega \backslash E$, since every Lipschitz function is approximately differentiable almost everywhere. But in fact, as presented in [ABO03], in view of [Fed69, Thm.3.1.8] (see also [GMS98, Thm.3, §3.1.4]), we can take, in the above, $E$ to be exactly the set of the points of non-approximate differentiability of $u$. By using the notation $N_{y}=u^{-1}(y) \backslash E$, we have thus arrived to the following coarea formula

$$
\int_{A} a(x)\left|u^{\sharp} \omega(x)\right| \mathrm{d} x=\int_{M}\left(\int_{N_{y} \cap A} a(x) \mathrm{d} \mathcal{H}^{p-m}(x)\right) \mathrm{d} \mathcal{H}^{m}(y),
$$

and also, by the same argument as before, viewing $N_{y}$ as a rectifiable current of constant multiplicity 1 , we deduce the representation formula

$$
\star u^{\sharp}(\rho \omega)=\int_{M}^{\star} \rho(y) \llbracket N_{y} \rrbracket \mathrm{d} \mathcal{H}^{m}(y),
$$

where the maps $a(\cdot)$ and $\rho$ satisfy the same properties as before.

2.4. The distributional Jacobian. If $u$ is a bounded map in $\mathrm{W}^{1, n}\left(\Omega, \mathbb{R}^{n+1}\right)$, the distributional Jacobian of $u$ is defined as the $(n+1)$-form on $\Omega$ given, in the distributional sense, by

$$
\mathrm{J} u=\frac{1}{n+1} \mathrm{~d}\left(u^{\sharp} \omega_{0}\right),
$$

where $\omega_{0}$ represents the volume form of the sphere $\mathbb{S}^{n}$. Since we have $\omega_{0}(y)=\sum_{i=1}^{n+1}(-1)^{i-1} y_{i} \widehat{\mathrm{d} y_{i}}$, for every $y \in \mathbb{S}^{n}$, where $\widehat{\mathrm{d} y_{i}} \equiv \mathrm{d} y_{1} \wedge \ldots \mathrm{d} y_{i-1} \wedge \mathrm{d} y_{i+1} \wedge \ldots \mathrm{d} y_{n+1}$, it means that

$$
\mathrm{J} u=\frac{1}{n+1} \mathrm{~d}\left(\sum_{\alpha \in I(n, p)} \operatorname{det}\left[\partial_{\alpha_{1}} u, \ldots, \partial_{\alpha_{n}} u, u\right] \mathrm{d} x_{\alpha_{1}} \wedge \cdots \wedge \mathrm{d} x_{\alpha_{n}}\right)
$$

The paper [Alb05] presents a review of the distributional Jacobian. We recall here a couple of basic properties of $\mathrm{J} u$. First of all, if $u$ is more regular, say it belongs to $\mathrm{W}^{1, n+1}\left(\Omega, \mathbb{R}^{n+1}\right)$, then

$$
\mathrm{J} u=\sum_{\alpha \in I(n+1, p)} \operatorname{det}\left[\partial_{\alpha_{1}} u, \ldots, \partial_{\alpha_{n+1}} u\right] \mathrm{d} x_{\alpha_{1}} \wedge \cdots \wedge \mathrm{d} x_{\alpha_{n+1}},
$$


so if $p=n+1$, than it is simply $\operatorname{det}(\mathrm{D} u) \mathrm{d} x$. This explains the choice of the factor $1 /(n+1)$ in the definition. Secondly, if $u \in \mathrm{W}^{1, n+1}\left(\Omega, \mathbb{S}^{n}\right)$, the partial derivatives $\partial_{1} u, \ldots, \partial_{n+1} u$ being linearly dependent pointwise, we necessarily have $\mathrm{J} u=0$.

A useful remark, given by [ABO03, Prop.7.9], is that, in the identity (17), we can replace $\omega_{0}$ by any differential $n$-form $\eta=\rho \omega_{0}$ with $\rho: \mathbb{S}^{n} \rightarrow \mathbb{R}$ a smooth function with average equal to 1 . Indeed, the integral over $\mathbb{S}^{n}$ of the $n$-form $(1-\rho) \omega_{0}$ equals zero, which implies that it is an exact form on $\mathbb{S}^{n}$ (see for example [Laf96, Theorem VII.B.6]). Then the conclusion follows, since the exterior derivative $\mathrm{d}$ commutes with the pullback and $\mathrm{d} \circ \mathrm{d}=0$.

The distributional Jacobian Ju describes in some sense the topological singularities of the map $u$. To exemplify, concerning $u \in \mathrm{W}^{1, n}\left(\Omega \subset \mathbb{R}^{p}, \mathbb{S}^{n}\right)$, the following are true:

(i) In the case $p=n+1$, the map $u$ can be approximated, in the $\mathrm{W}^{1, n}$ norm, by a sequence $\left\{u_{j}\right\} \subset \mathrm{C}^{\infty}\left(\Omega, \mathbb{S}^{n}\right)$ if and only if $\mathrm{J} u=0$.

(ii) There exists a sequence $\left\{u_{j}\right\} \subset \mathrm{C}^{\infty}\left(\Omega \backslash \Gamma_{j}, \mathbb{S}^{n}\right)$ such that $u_{j} \rightarrow u$ in $\mathrm{W}^{1, n}$, where $\Gamma_{j}=\partial\left(u_{j}^{-1}(y)\right)$, for $\mathcal{H}^{n}$-almost every $y \in \mathbb{S}^{n}$.

(iii) If the mapping $u$ is continuous outside a $(p-n-1)$-dimensional submanifold $S$, then $\star \mathrm{J} u=\frac{\sigma_{n+1}}{n+1} \operatorname{deg}(u, S) \llbracket S \rrbracket$.

(iv) In general, $\star \mathrm{J} u=(-1)^{p-n} \frac{\sigma_{n+1}}{n+1} \partial \llbracket N_{y} \rrbracket$ for $\mathcal{H}^{n}$-almost all $y \in \mathbb{S}^{n}$.

The property (i) is the main result of [Bet90] ([Remark 2]). The approximation result (ii) can be proved by following the same argument used in [Bou07, Section 5] to prove the density of the class $\mathcal{R}$ in $\mathrm{W}^{s, q}\left(\mathbb{S}^{N}, \mathbb{S}^{1}\right)$ (for $s, q \geqslant 1$ with $1 \leqslant s q<2$, [Bou07, Theorem 2]). The property (iii) is stated in [ABO03, Subsection 3.7] and it was previously proved, for $p=n+1$ in [BCL86, Theorem B.2, Remark B.2], for $n=2$ and $S$ a $(p-3)$-dimensional disk in [Pak01, Corollary 1], and for $p=3, n=1$ in [JS02, Example 3.4]. And finally, (iv) is one of the conclusions of [ABO03, Theorem 3.8], and we detail below its proof.

We have seen that the Jacobian of $u$ is given by $\mathrm{J} u=\frac{1}{n+1} \mathrm{~d}\left(u^{\sharp}\left(\rho \omega_{0}\right)\right)$, where $\rho$ is any real smooth function on $\mathbb{S}^{n}$ with average equal to 1 , and $\omega_{0}$ is the volume form on $\mathbb{S}^{n}$. Hence, by the properties of the $\star$ operator, we have

$$
\star \mathrm{J} u=\frac{(-1)^{m}}{n+1} \partial\left(\star u^{\sharp}\left(\rho \omega_{0}\right)\right) .
$$

We fix a point $z \in \mathbb{S}^{n}$ where $y \mapsto \llbracket N_{y} \rrbracket$ is weak-^ approximately continuous. This is in fact true for $\mathcal{H}^{n}$-almost all $z$, because, as we have seen, the map $y \mapsto \llbracket N_{y} \rrbracket$ is weak- $\star \mathcal{H}^{n}$-measurable. So, if we consider $\left\{\eta_{i}\right\}$ a dense countable subset of $\mathcal{D}^{m}(\Omega)$, then, for every $\eta \in \mathcal{D}^{m}(\Omega)$, $y \mapsto \llbracket N_{y} \rrbracket(\eta)$ is $\mathcal{H}^{n}$-measurable, hence $\mathcal{H}^{n}$-almost everywhere approximately continuous. Thus, for $\mathcal{H}^{n}$ almost all $z$, the map $y \mapsto \llbracket N_{y} \rrbracket\left(\eta_{i}\right)$ is approximately continuous in $z$, for every $i$, and, by density, it follows that all such points $z$ are actually points of weak-^ approximate continuity of $\llbracket N_{y} \rrbracket$.

Let $\rho_{i}: \mathbb{S}^{n} \rightarrow \mathbb{R}$ be a sequence of positive smooth functions with compact support which approximate the Dirac measure at $z$, such that

$$
\begin{gathered}
\operatorname{supp}\left(\rho_{i}\right)=B\left(z, r_{i}\right) \cap \mathbb{S}^{n}, \quad \int_{\mathbb{S}^{n}} \rho_{i} \mathrm{~d} \mathcal{H}^{n}=1, \quad \text { and } \\
\sup _{i} \sup _{x}\left\{\left|\rho_{i}(x)\right| \mathcal{H}^{n}\left(\operatorname{supp}\left(\rho_{i}\right)\right)\right\}=: \alpha<\infty .
\end{gathered}
$$


Then, using the formula (16), we have that, for any $\eta \in \mathcal{D}^{m}(\Omega)$,

$$
\begin{aligned}
\left|\star u^{\sharp}\left(\rho_{i} \omega_{0}\right)(\eta)-\llbracket N_{z} \rrbracket(\eta)\right| & =\left|\int_{\mathbb{S}^{n}} \rho_{i}(y) \llbracket N_{y} \rrbracket(\eta) \mathrm{d} \mathcal{H}^{n}(y)-\llbracket N_{z} \rrbracket(\eta)\right| \\
& \leqslant \int_{\mathbb{S}^{n}} \rho_{i}(y)\left|\llbracket N_{y} \rrbracket(\eta)-\llbracket N_{z} \rrbracket(\eta)\right| \mathrm{d} \mathcal{H}^{n}(y) \\
& \leqslant \alpha f_{B\left(z, r_{i}\right) \cap \mathbb{S}^{n}}\left|\llbracket N_{y} \rrbracket(\eta)-\llbracket N_{z} \rrbracket(\eta)\right| \mathrm{d} \mathcal{H}^{n}(y),
\end{aligned}
$$

which converges to 0 when $r_{i}$ tends to 0 , hence $\star u^{\sharp}\left(\rho_{i} \omega_{0}\right)$ converges, in the weak- $\star$ topology, to $\llbracket N_{z} \rrbracket$. Since the boundary operator is continuous, this implies that

$$
\partial \llbracket N_{z} \rrbracket=\frac{(-1)^{m}(n+1)}{\sigma_{n+1}} \star \mathrm{J} u,
$$

noting that we have to use $\sigma_{n+1} \rho_{i}$ in place of $\rho$ in the relation (18).

We can now state the main result of the paper.

Theorem 1. Let $\Omega$ be a bounded and connected smooth open set in $\mathbb{R}^{m+n}$, and suppose $\Gamma$ is the boundary of a rectifiable current in $\Omega$, of dimension $m$ and finite mass. Consider the following class of admissible functions

$$
\mathcal{E}(\Gamma):=\left\{u \in \mathrm{W}^{1, n}\left(\Omega, \mathbb{S}^{n}\right) ; \star \mathrm{J} u=\frac{\sigma_{n+1}}{n+1} \Gamma\right\},
$$

To each map u in $\mathcal{E}(\Gamma)$, we associate the weighted energy

$$
E_{\Gamma}(u, a)=\int_{\Omega} a(x)|\mathrm{D} u(x)|^{n} \mathrm{~d} x,
$$

for any strictly positive, continuous function $a(\cdot)$ on $\Omega$, with $\inf a(\cdot)>0$. Then, the minimum energy of $u$ is given by

$$
\inf _{u \in \mathcal{E}(\Gamma)} E_{\Gamma}(u, a)=n^{n / 2} \sigma_{n+1} \inf _{M \in \mathcal{C}(\Gamma)} M(M\llcorner a),
$$

where the infimum in the right-hand side is taken over the following class of integral currents,

$$
\mathcal{C}(\Gamma):=\left\{M \in \mathcal{R}_{m}\left(\mathbb{R}^{m+n}\right) ; M(M)<\infty, \partial M=\Gamma\right\} .
$$

\section{THE PROOF OF THEOREM 1.}

The first step is the construction of the dipole which will be used in the proof of the upper bound of the energy. This intermediate result, given in Lemma 1, follows the one in [BCL86, Section VIII, 1. The Upper Bound], concerned with the case $m=2, n=1$. In order to take into account a continuous weight function, we define the following modified spherical Hausdorff measure.

Definition 1. For any positive $\mathcal{L}^{p}$-measurable function $w(\cdot)$ on $\mathbb{R}^{p}$, let $\mathcal{H}_{w}^{m}$ denote the outer measure on $\mathbb{R}^{p}$ defined by

$$
\begin{array}{r}
\mathcal{H}_{w}^{m}(A)=\sup _{\delta>0} \inf \left\{\sum_{k=1}^{N} \frac{p^{m / p} \sigma_{m}}{m \sigma_{p}^{m / p}}\|w\|_{L^{p / m}\left(D_{k}\right)}: D_{k} \subset \mathbb{R}^{p}\right. \text { open balls } \\
\text { that cover } \left.A, \operatorname{diam} D_{k}<\delta\right\},
\end{array}
$$

for every $A \subset \mathbb{R}^{p}$. 
Since $\mathcal{H}_{w}^{m}$ is obtained, like the usual Hausdorff measure, via the Carathéodory construction, it is indeed a (metric) outer measure on $\mathbb{R}^{p}$, and the following result shows the connection between $\mathcal{H}_{w}^{m}$ and $\mathcal{H}^{m}$.

Proposition 1. If $w(\cdot)$ is a continuous positive function, then

$$
\mathcal{H}_{w}^{m}\left\llcorner A=w(\cdot) \mathcal{H}^{m}\llcorner A,\right.
$$

for any $\mathcal{H}^{m}$-rectifiable set $A \subset \mathbb{R}^{p}$.

Proof. First we see that, if $w(\cdot)$ is constant, then the above equality is satisfied, because, by [Fed69, Theorem 3.2.26], the Hausdorff and spherical measures coincide on $\mathcal{H}^{m}$-rectifiable sets. Next, remark that the two measures, $\mathcal{H}_{w}^{m}\left\llcorner A\right.$ and $w(\cdot) \mathcal{H}^{m}\llcorner A$, are both Radon measures since, by hypothesis, $\mathcal{H}^{m}(A)<\infty$, and so, also $\mathcal{H}_{w}^{m}(A)<\infty$. The sets of null $\mathcal{H}_{w}^{m}$ and $\mathcal{H}^{m}$-measure coincide, so it is enough to prove

$$
\mathcal{H}_{w}^{m}(A)=\int_{A} w(x) \mathrm{d} \mathcal{H}^{m}(x),
$$

for any set $A$ that is Borel $\mathcal{H}^{m}$-rectifiable and compact.

We will apply the Vitali-Besicovitch covering theorem (see [AFP00, Theorem 2.19]), to cover $A$, up to a set of small $\mathcal{H}^{m}$-measure, with a disjoint family of small balls. For this, first, for any $\varepsilon>0$, we choose $\gamma>0$ so that $w(\cdot)$, which is uniformly continuous on $A$, satisfies $|w(x)-w(y)| \leqslant \varepsilon$, whenever $|x-y| \leqslant \gamma$. Then, for any $\delta>0$, we can find a finite number of balls such that

$$
\mathcal{H}^{m}\left(A \backslash \bigcup_{j=1}^{N} B_{\gamma_{j}}\left(x_{j}\right)\right) \leqslant \delta, \quad \text { where } \quad \gamma_{j} \leqslant \gamma, x_{j} \in A, \forall j=1, \ldots, N .
$$

With this, we have

$$
\int_{A} w(x) \mathrm{d} \mathcal{H}^{m}(x) \leqslant \delta \sup _{A} w(\cdot)+\varepsilon \mathcal{H}^{m}(A)+\sum_{j=1}^{N} w\left(x_{j}\right) \mathcal{H}^{m}\left(B_{\gamma_{j}}\left(x_{j}\right) \cap A\right)
$$

and, knowing that $w\left(x_{j}\right) \mathcal{H}^{m}\left\llcorner A=\mathcal{H}_{w\left(x_{j}\right)}^{m}\left\llcorner A\right.\right.$, for all $j$, and $w\left(x_{j}\right) \leqslant \varepsilon+w(x)$, for all $x \in B_{\gamma_{j}}\left(x_{j}\right)$, we arrive to

$$
\int_{A} w(x) \mathrm{d} \mathcal{H}^{m}(x) \leqslant \delta \sup _{A} w(\cdot)+2 \varepsilon \mathcal{H}^{m}(A)+\mathcal{H}_{w}^{m}(A) .
$$

On the other hand, we get, in the same manner, that

$$
\mathcal{H}_{w}^{m}(A) \leqslant \delta \sup _{A} w(\cdot)+2 \varepsilon \mathcal{H}^{m}(A)+\int_{A} w(x) \mathrm{d} \mathcal{H}^{m}(x),
$$

so, by taking $\varepsilon, \delta \rightarrow 0$, we have obtained the desired equality.

We assume, from now on, the hypotheses of Theorem 1. Following the previous lemma, we could define a new mass $\mathbb{M}_{a}$ on $\mathcal{R}_{m}\left(\mathbb{R}^{m+n}\right)$ by substituting $\mathcal{H}^{m}$ for $\mathcal{H}_{a}^{m}$ in the integral representation of the mass $\mathbb{M}$, see (9) in section 2. Specifically, for a current $T \in \mathcal{R}_{m}\left(\mathbb{R}^{m+n}\right)$ associated to the $\mathcal{H}^{m}$-rectifiable set $\widetilde{T}$ and the multiplicity $\theta_{T}$, we set

$$
\mathbb{M}_{a}(T)=\int_{\widetilde{T}} \theta_{T}(x) \mathcal{H}_{a}^{m} .
$$

With this, formula (20) can be written as

$$
\inf _{u \in \mathcal{E}(\Gamma)} E_{\Gamma}(u, a)=n^{n / 2} \sigma_{n+1} \inf _{M \in \mathcal{C}(\Gamma)} \mathbb{M}_{a}(M),
$$


which is in agreement with the observation in [BCL86] that different generalizations of the original problem should all yield the same kind of formula for the minimum energy, provided that the distance, in this case the measure, is properly defined. The same approach was taken in [Mil05], where the Euclidean distance in $\mathbb{R}^{3}$ was replaced by dist ${ }_{a}$ whose definition we specified in the Introduction, formula (7). Considering the proposition below, it is clear that Theorem 1 gives, for the case $m=1, n=2$, the same formula, as does [Mil05, Theorem 1.1], for $a(\cdot)$ continuous.

Proposition 2. Suppose $\Gamma$ is a polyhedral current. Then, in the formula (20) of Theorem 1, we can assume that the currents $M$ are polyhedral, i.e.,

$$
\inf \left\{M(M\llcorner a): M \in \mathcal{C}(\Gamma)\}=\inf \left\{M\left(M\llcorner a): M \in \mathcal{C}(\Gamma) \cap \mathcal{P}_{m}\left(\mathbb{R}^{m+n}\right)\right)\right\}\right.
$$

Proof. We need to show that, given $M \in \mathcal{R}_{m}\left(\mathbb{R}^{m+n}\right)$, with finite mass and boundary $\partial M=\Gamma$, we can find, for every $\varepsilon>0$, a polyhedral current $P$, that has the same boundary, and which satisfies

$$
M(P\llcorner a) \leqslant M(M\llcorner a)+\varepsilon .
$$

It suffices to show this for $a(\cdot) \equiv 1$, as the general case follows by applying the VitaliBesicovitch theorem as in the precedent proof.

By the approximation theorem [Fed69, Theorem 4.2.22], there exists a polyhedral current $P_{0} \in \mathcal{P}_{m}\left(\mathbb{R}^{m+n}\right)$, and two rectifiable currents $R$ and $Q$, of dimension $m$ and $m+1$, respectively, such that

$$
M=P_{0}+R+\partial Q, \quad \text { with } \quad \mathbb{M}(R) \leqslant \varepsilon / 2, \quad \text { and } \quad M\left(P_{0}\right) \leqslant M+\varepsilon / 2 .
$$

Moreover, by the deformation theorem [Fed69, Theorem 4.2.9], we can write $R$ as the sum between a polyhedral current $P_{1}$ and the boundary $\partial S$ of a $(m+1)$-dimensional integral current $S$. So, if we take $P=P_{0}+P_{1}$, we have

$$
M=P+\partial(Q+S), \quad \text { with } \quad M(P) \leqslant M\left(P_{0}\right)+M(R) \leqslant \varepsilon,
$$

and $\partial P=\partial M=\Gamma$.

The following lemma gives the analogue of the dipole introduced in [BCL86].

Lemma 1. Suppose $E \equiv E_{r}\left(\xi_{0}, \tau_{E}\right)$ is an oriented disk of dimension $m$ in $\Omega$. Then, for any $\delta>0$, and any fixed point $y_{0} \in \mathbb{S}^{n}$, there exists a map $u \in \mathcal{E}(\llbracket \partial E \rrbracket)$, that is locally Lipschitz in $\Omega \backslash \partial E$, constantly equal to $y_{0}$ outside the ball $B_{r}\left(\xi_{0}\right)$ in $\mathbb{R}^{m+n}$, and whose energy satisfies the following inequality

$$
\int_{\Omega} a(x)|\mathrm{D} u(x)|^{n} \mathrm{~d} x \leqslant n^{n / 2} \sigma_{n+1} \mathcal{H}_{a}^{m}(E)+\delta .
$$

Proof. Without loss of generality, we may restrict to the particular case where

$$
\begin{gathered}
E=\left\{(x, 0) \in \mathbb{R}^{m} \times \mathbb{R}^{n} \text { with }|x| \leqslant r\right\}, \\
\tau_{E}=e_{1}^{(m+n)} \wedge \cdots \wedge e_{m}^{(m+n)} \in \mathbb{R}^{m} \times \mathbb{R}^{n}, \quad \text { and } y_{0}=(0,1) \in \mathbb{R}^{n} \times \mathbb{R},
\end{gathered}
$$

because the general case will then follow through an isometry of $\mathbb{R}^{n+m}$, and a rotation of $\mathbb{S}^{n}$. 
The map $u$ will be constructed in two steps, just as described in [BCL86]. The first step is to take a function $v: \mathbb{R}^{n} \rightarrow \mathbb{S}^{n}$ that satisfies the following properties:

$$
v(\cdot) \text { is Lipschitz on } \mathbb{R}^{n} \text { and constant outside a small ball, }
$$

$$
\begin{gathered}
\operatorname{deg} v(\cdot)=1, \text { and } \\
\int_{\mathbb{R}^{n}}|\mathrm{D} v(x)|^{n} \mathrm{~d} x \leqslant n^{n / 2} \sigma_{n+1}+\eta,
\end{gathered}
$$

for a given $\eta>0$. For example, we can consider the function from [BC83, proof of Lemma 2] (see also [Mil05, Lemma 3.2]), which, in our case, becomes

$$
v(\varepsilon, x)= \begin{cases}\frac{1}{\varepsilon^{4}+|x|^{2}}\left(2 \varepsilon^{2} \bar{x},|x|^{2}-\varepsilon^{4}\right) & |x| \leqslant \varepsilon \\ \left(\frac{\bar{x}}{|x|} A(|x|), \sqrt{1-A^{2}(|x|)}\right) & |x| \in[\varepsilon, 2 \varepsilon], \\ (0,1) \in \mathbb{R}^{n} \times \mathbb{R} & |x| \geqslant 2 \varepsilon\end{cases}
$$

where $\bar{x}:=\left((-1)^{n+1} x_{1}, x_{2}, \ldots, x_{n}\right)$ is used to control the sign of $\operatorname{deg} v(\varepsilon, \cdot)$, the map $A(\cdot)$ is an affine map chosen such that $v(\varepsilon, \cdot)$ is continuous, that is,

$$
A(r):=-\frac{2}{\varepsilon^{2}+1} r+\frac{4 \varepsilon}{\varepsilon^{2}+1}, \text { for } r>0
$$

and $\varepsilon>0$ is a small parameter that we will conveniently fix later.

The meaning of $\operatorname{deg} v(\varepsilon, \cdot)$ is that of the degree of $v(\varepsilon, \cdot)$ which is viewed as a map from the sphere $\mathbb{S}^{n}$ to itself, obtained by identifying $\mathbb{R}^{n} \cup\{\infty\}$ and $\mathbb{S}^{n}$ through the use of the stereographic projection $\pi$. So $\operatorname{deg} v(\varepsilon, \cdot)$ is given by

$$
\begin{aligned}
\operatorname{deg} v(\varepsilon, \pi(\cdot)) & =\frac{1}{\sigma_{n+1}} \int_{\mathbb{S}^{n}} \operatorname{det}\left[v(\varepsilon, \pi(y)), \partial_{y_{1}} v(\varepsilon, \pi(y)), \ldots, \partial_{y_{n}} v(\varepsilon, \pi(y))\right] \mathrm{d} \mathcal{H}^{n}(y) \\
& =\frac{1}{\sigma_{n+1}} \int_{\mathbb{R}^{n}} \operatorname{det}\left[v(\varepsilon, x), \partial_{x_{1}} v(\varepsilon, x), \ldots, \partial_{x_{n}} v(\varepsilon, x)\right] \mathrm{d} x,
\end{aligned}
$$

where $y_{1} \ldots, y_{n}$ are the coordinates of the point $y \in \mathbb{S}^{n}$ in an orthonormal basis of $\mathrm{T}_{y} \mathbb{S}^{n}$.

The function $v(\varepsilon, \cdot)$ clearly satisfies $(22)$, and we next check that, provided $\varepsilon$ is sufficiently small, properties (23) and (24) are also verified. Since

$$
\operatorname{det}\left[v, \partial_{x_{1}} v, \ldots, \partial_{x_{n}} v\right](\varepsilon, x)=\left\{\begin{array}{l}
\left(\frac{2 \varepsilon^{2}}{\varepsilon^{4}+|x|^{2}}\right)^{n}, \quad|x| \leqslant \varepsilon \\
\frac{2}{\varepsilon^{2}+1} \times \frac{A^{n-1}(|x|)}{|x|^{n-1} \sqrt{1-A^{2}(|x|)}}, \quad|x| \in[\varepsilon, 2 \varepsilon],
\end{array}\right.
$$

we have, after the appropriate change of variables, that

$$
\operatorname{deg} v(\varepsilon, \cdot)=\frac{\sigma_{n}}{\sigma_{n+1}}\left(2^{n} \int_{0}^{1 / \varepsilon} \frac{r^{n-1}}{\left(1+r^{2}\right)^{n}} \mathrm{~d} r+\int_{0}^{2 \varepsilon /\left(\varepsilon^{2}+1\right)} \frac{r^{n-1}}{\sqrt{1-r^{2}}} \mathrm{~d} r\right) .
$$

We notice that the derivative with respect to $\varepsilon$ of $\operatorname{deg} v(\varepsilon, \cdot)$ vanishes on the interval $(0,1)$. Since the degree depends continuously on $\varepsilon$, we find, after another change of variables, that

$$
\operatorname{deg} v(\varepsilon, \cdot)=\operatorname{deg} v(1, \cdot)=\frac{\sigma_{n}}{2 \sigma_{n+1}}\left(2^{n} \int_{0}^{1} \frac{t^{n / 2-1}}{(1+t)^{n}} \mathrm{~d} t+\int_{0}^{1} \frac{t^{n / 2-1}}{(1-t)^{1 / 2}} \mathrm{~d} t\right) .
$$


The second integral is an Euler integral, and its value is

$$
\int_{0}^{1} t^{n / 2-1}(1-t)^{1 / 2-1} \mathrm{~d} t=\mathrm{B}\left(\frac{n}{2}, \frac{1}{2}\right)=\frac{\Gamma\left(\frac{n}{2}\right) \Gamma\left(\frac{1}{2}\right)}{\Gamma\left(\frac{n}{2}+\frac{1}{2}\right)}
$$

where $\mathrm{B}(\cdot, \cdot)$ and $\Gamma(\cdot)$ are the classical Beta and Gamma functions, respectively. For the first integral in (26), we consider the hypergeometric function in its Euler integral representation, that is,

$$
\mathrm{F}(\alpha, \beta ; \gamma ; \xi)=\frac{\Gamma(\gamma)}{\Gamma(\beta) \Gamma(\gamma-\beta)} \int_{0}^{1} t^{\beta-1}(1-t)^{\gamma-\beta-1}(1-\xi t)^{-\alpha} \mathrm{d} t
$$

(see for example [AAR99, Theorem 2.2.1]; here $\alpha, \beta, \gamma>0$ and $\xi \in \mathbb{R} \backslash\{0\}$ ), and then use Kummer's formula

$$
\mathrm{F}(\alpha, \beta ; \alpha-\beta+1 ;-1)=\frac{\Gamma(\alpha-\beta+1) \Gamma\left(\frac{\alpha}{2}+1\right)}{\Gamma(\alpha+1) \Gamma\left(\frac{\alpha}{2}-\beta+1\right)}
$$

(see [AAR99, Corollary 3.1.2]). This gives us

$$
\int_{0}^{1} t^{n / 2-1}(1+t)^{-n} \mathrm{~d} t=\frac{\Gamma\left(\frac{n}{2}+1\right) \Gamma\left(\frac{n}{2}\right)}{\Gamma(n+1)},
$$

and, using the property $\Gamma(z) \Gamma\left(z+\frac{1}{2}\right)=2^{1-2 z} \sqrt{\pi} \Gamma(2 z)$ satisfied by the Gamma function, we conclude that (23) is verified, that is,

$$
\operatorname{deg} v(\varepsilon, \cdot)=1, \quad \text { for any } \varepsilon \in(0,1) .
$$

In order to verify $(24)$, we compute the differential of $v(\varepsilon, \cdot)$. We have that

$$
|\mathrm{D} v(\varepsilon, x)|= \begin{cases}n^{1 / 2} \frac{2 \varepsilon^{2}}{\varepsilon^{4}+|x|^{2}} & |x| \leqslant \varepsilon \\ \left(\frac{4}{\left(\varepsilon^{2}+1\right)^{2}} \times \frac{1}{1-A^{2}(|x|)}+\frac{(n-1) A^{2}(|x|)}{|x|^{2}}\right)^{1 / 2} & |x| \in[\varepsilon, 2 \varepsilon] .\end{cases}
$$

Thus,

$$
\int_{|x| \leqslant \varepsilon}|\mathrm{D} v(\varepsilon, x)|^{n} \mathrm{~d} x=n^{n / 2} \sigma_{n} \int_{0}^{\varepsilon}\left(\frac{2 \varepsilon^{2}}{\varepsilon^{4}+r^{2}}\right)^{n} r^{n-1} \mathrm{~d} r=n^{n / 2} \sigma_{n} 2^{n} \times I(\varepsilon),
$$

where $I(\varepsilon)$ is the first integral in (25). From the fact that $\operatorname{deg} v(\varepsilon, \cdot)=1$, and recalling (25), we can deduce that

for every $\varepsilon \in(0,1)$, and so

$$
I(\varepsilon)=\frac{\sigma_{n+1}}{2^{n} \sigma_{n}}-\frac{1}{2^{n}} \int_{0}^{2 \varepsilon /\left(\varepsilon^{2}+1\right)} \frac{r^{n-1}}{\sqrt{1-r^{2}}} \mathrm{~d} r,
$$

$$
\lim _{\varepsilon \rightarrow 0} \int_{|x| \leqslant \varepsilon}|\mathrm{D} v(\varepsilon, x)|^{n} \mathrm{~d} x=n^{n / 2} \sigma_{n+1} .
$$

On the other hand,

$$
\begin{aligned}
\int_{\varepsilon \leqslant|x| \leqslant 2 \varepsilon} & |\mathrm{D} v(\varepsilon, x)|^{n} \mathrm{~d} x \\
& =2^{n} \sigma_{n} \int_{\varepsilon}^{2 \varepsilon}\left(\frac{1}{\left(\varepsilon^{2}+1\right)^{2}-4(2 \varepsilon-r)^{2}}+(n-1) \frac{(2 \varepsilon-r)^{2}}{r^{2}\left(\varepsilon^{2}+1\right)}\right)^{n / 2} r^{n-1} \mathrm{~d} r \\
& \leqslant 2^{2 n} \sigma_{n} \varepsilon^{n}\left(\frac{1}{\left(1-\varepsilon^{2}\right)^{2}}+\frac{n-1}{\varepsilon^{2}+1}\right)^{n / 2}
\end{aligned}
$$


which tends to 0 when $\varepsilon \rightarrow 0$. So, we can choose $\varepsilon>0$ sufficiently small for (24) to be verified, and for this function $v(\varepsilon, \cdot)$ we will just use the notation $v(\cdot)$.

The second step is to define, for every $k \geqslant 1$, the function $u: \Omega \backslash \partial E \rightarrow \mathbb{S}^{n}$ by

$$
u(\tilde{x}, x)= \begin{cases}v\left(\frac{k x}{r-|\tilde{x}|}\right) & (\tilde{x}, x) \in\left(\operatorname{pr}_{\mathbb{R}^{m}} E \times \mathbb{R}^{n}\right) \cap \Omega \\ (0,1) \in \mathbb{R}^{n} \times \mathbb{R} & \text { elsewhere } .\end{cases}
$$

We compute the differential of $u$ to get

$$
|\mathrm{D} u(\tilde{x}, x)|=\left(\frac{k}{r-|\tilde{x}|}\right)\left[\left(\frac{|x|}{r-|\tilde{x}|}\right)^{2}+1\right]^{1 / 2} \times\left|\mathrm{D} v\left(\frac{k x}{r-|\tilde{x}|}\right)\right|,
$$

if $(\tilde{x}, x) \in \operatorname{pr}_{\mathbb{R}^{m}} E \times \mathbb{R}^{n}$. For $k$ sufficiently large, and $\eta$ conveniently chosen, we have

$$
\begin{aligned}
\int_{\Omega} a(x) \mid & \left.\mathrm{D} u(x)\right|^{n} \mathrm{~d} x \\
& =\int_{\operatorname{pr}_{\mathbb{R}} m E \cap \Omega}\left(\int_{\mathbb{R}^{n} \cap \Omega} a\left(\tilde{x}, \frac{y(r-|\tilde{x}|)}{k}\right)\left[\left(\frac{|y|}{k}\right)^{2}+1\right]^{n / 2} \times|\mathrm{D} v(y)|^{n} \mathrm{~d} y\right) \mathrm{d} \tilde{x} \\
& \leqslant n^{n / 2} \sigma_{n+1} \int_{\operatorname{pr}_{\mathbb{R} m} E \cap \Omega} a(\tilde{x}, 0) \mathrm{d} \tilde{x}+\delta=n^{n / 2} \sigma_{n+1} \mathcal{H}_{a}^{m}(E)+\delta,
\end{aligned}
$$

so inequality (21) is satisfied.

Since the map $u$ belongs to $\mathrm{W}_{\text {loc }}^{1, n}\left(\Omega, \mathbb{S}^{n}\right)$, and is locally Lipschitz outside $\partial E$, we know that

$$
\star \mathrm{J} u=\frac{\sigma_{n+1}}{n+1} \operatorname{deg}(u, \partial E) \llbracket \partial E \rrbracket,
$$

where the degree of $u$ along the curve $\partial E$ means the degree of the restriction of $u$ to the boundary of any disk $D \subset \mathbb{R}^{m+n}$ of dimension $(n+1)$, that intersects transversally $\partial E$ in only one point, and whose orientation $\tau_{D}$ is such that

$$
\tau_{\partial E} \wedge \tau_{D}=e_{1}^{(m+n)} \wedge \cdots \wedge e_{m+n}^{(m+n)},
$$

$\tau_{\partial E}$ being the orientation of the boundary $\partial E$ induced by that of $E$. If we choose

$$
D=\left\{\xi=\tilde{x}_{1} e_{1}^{(m+n)}+x_{1} e_{m+1}^{(m+n)}+\cdots+x_{n} e_{m+n}^{(m+n)} \text { with }\left|\xi-r e_{1}^{(m+n)}\right| \leqslant r\right\},
$$

then it can be easily seen that $\operatorname{deg}(u, \partial D)=\operatorname{deg} v(\cdot)=1$, and, consequently, the map $u$ belongs to the class $\mathcal{E}(\llbracket \partial E \rrbracket)$.

For passing from the boundary of a disk to the general case of the boundary of a rectifiable current, we will need the following result, which is a variant of [ABO03, Corollary 7.13].

Theorem 2. Let $T$ be a current in $\mathcal{R}_{m}(\Omega)$ with finite mass, and suppose that $a(\cdot): \Omega \rightarrow(0, \infty)$ is a bounded continuous function. Given $\rho(\cdot)$ a strictly positive function on $\Omega$, and $s>1$, there exist, for any $\varepsilon>0$, a rectifiable current $R \in \mathcal{R}_{m}(\Omega)$, and finitely many m-dimensional disks $E_{r_{i}}\left(x_{i}\right) \subset \Omega$ of radii $r_{i} \leqslant \rho\left(x_{i}\right)$, which satisfy the following properties:
(a) $\partial T=\sum_{i} \partial \llbracket E_{r_{i}}\left(x_{i}\right) \rrbracket+\partial R$;
(b) $\sum_{i} \mathcal{H}_{a}^{m}\left(E_{r_{i}}\left(x_{i}\right)\right) \leqslant \frac{1+\varepsilon}{s^{m}} M(T\llcorner a)$;
(c) $M\left(R\llcorner a) \leqslant\left(1-\frac{1}{s^{m+1}}\right) M(T\llcorner a)\right.$;
(d) the balls $\bar{B}_{s r_{i}}\left(x_{i}\right)$ are pairwise disjoint and contained in $\Omega$. 
Proof. This result is a direct consequence of [ABO03, Theorem 7.12] (which, in turn, is proved using the approximation of integral flat currents by integral polyhedral currents - [Fed69, Thm. 4.2.22].)

We begin, just like in the proof of proposition 1, by applying the Vitali-Besicovitch covering theorem. Since $a(\cdot)$ is uniformly continuous on the compact support of $T$, then, for any $\eta>0$, we can find $\gamma>0$ such that, $|a(x)-a(y)| \leqslant \eta$ whenever $|x-y| \leqslant \gamma$, and, considering $T$ given by the integral representation (8), we cover $\widetilde{T}$, up to a set of small $\mathcal{H}^{m}$-measure, by a finite and disjoint family of small balls

$$
\mathcal{H}^{m}\left(\widetilde{T} \backslash \bigcup_{j=1}^{p} B_{\gamma_{j}}\left(\xi_{j}\right)\right) \leqslant \lambda, \quad \text { where } \quad \lambda>0, \gamma_{j} \leqslant \gamma, \xi_{j} \in \widetilde{T} .
$$

The theorem mentioned above states that the currents $T\left\llcorner B_{\gamma_{j}}\left(\xi_{j}\right)\right.$ can be approximated in the flat norm by finite sums $\sum_{i=1}^{p_{j}} \llbracket E_{\rho_{i j}}\left(x_{i j}\right) \rrbracket$ of $m$-dimensional disks with radii $\rho_{i j}<\rho\left(x_{i j}\right)$ and such that $\bar{B}_{\rho_{i j}}\left(x_{i j}\right)$ are pairwise disjoint and contained in $B_{\gamma_{j}}\left(x_{j}\right)$. This implies that, for any $\delta>0$, there exist rectifiable currents $R_{j} \in \mathcal{R}_{m}\left(B_{\gamma_{j}}\left(x_{j}\right)\right)$ and $Q_{j} \in \mathcal{R}_{m+1}\left(B_{\gamma_{j}}\left(x_{j}\right)\right)$, that satisfy

$$
\begin{gathered}
T\left\llcorner B_{\gamma_{j}}\left(\xi_{j}\right)=\sum_{i=1}^{p_{j}} \llbracket E_{\rho_{i j}}\left(x_{i j}\right) \rrbracket+R_{j}+\partial Q_{j}\right. \\
\mathbb{M}\left(R_{j}\right) \leqslant \delta M\left(T\left\llcorner B_{\gamma_{j}}\left(\xi_{j}\right)\right), \quad\right. \text { and } \\
\sum_{i=1}^{p_{j}} \mathcal{H}^{m}\left(E_{\rho_{i j}}\left(x_{i j}\right)\right) \leqslant(1+\delta) M\left(T\left\llcorner B_{\gamma_{j}}\left(\xi_{j}\right)\right), \forall j=1 \ldots, p .\right.
\end{gathered}
$$

We rescale the radii by taking $r_{i j}:=\rho_{i j} / s$, define $R_{0}:=T\llcorner A$, where the measurable set $A$ represents the difference $\widetilde{T} \backslash \bigcup_{j=1}^{p} B_{\gamma_{j}}\left(\xi_{j}\right)$, and consider the following $m$-dimensional rectifiable current

$$
R:=\sum_{j=1}^{p}\left[\sum_{i=1}^{p_{j}}\left(\llbracket E_{\rho_{i j}}\left(x_{i j}\right) \rrbracket-\llbracket E_{r_{i j}}\left(x_{i j}\right) \rrbracket\right)+R_{j}\right]+R_{0} .
$$

After summing the identities in (27), we obtain

$$
T=\sum_{j=1}^{p} \sum_{i=1}^{p_{j}} \llbracket E_{i j}\left(x_{i j}\right) \rrbracket+R+\sum_{j} \partial Q_{j}
$$

so we need only to apply the boundary operator, and reindex the finite number of disks $E_{r_{i j}}\left(x_{i j}\right)$, to get the first property required.

To arrive at the second one, we approximate $a(x)$ with $a\left(\xi_{j}\right)$ on each $B_{\gamma_{j}}\left(\xi_{j}\right)$, and use the inequalities in (29), that give us

$$
\begin{aligned}
\sum_{j=1}^{p} \sum_{i=1}^{p_{j}} \mathcal{H}_{a}^{m}\left(E_{r_{i j}}\left(x_{i j}\right)\right) & \leqslant \sum_{j=1}^{p} \sum_{i=1}^{p_{j}}\left(a\left(\xi_{j}\right)+\eta\right) \mathcal{H}^{m}\left(E_{r_{i j}}\left(x_{i j}\right)\right) \\
& \leqslant \frac{1+\delta}{s^{m}}(\mathbb{M}(T\llcorner a)+2 \eta \mathbb{M}(T)) .
\end{aligned}
$$


Then, using also the inequalities (28), and the fact that $A$ has small $\mathcal{H}^{m}$ measure, we see that

$$
\begin{aligned}
M(R\llcorner a) & =\sum_{j=1}^{p} \sum_{i=1}^{p_{j}}\left[\mathbb { M } \left(\llbracket E_{\rho_{i j}}\left(x_{i j}\right) \rrbracket\llcorner a)-M\left(\llbracket E_{r_{i j}}\left(x_{i j}\right) \rrbracket\llcorner a)\right]\right.\right. \\
& +\sum_{j=1}^{p} \mathbb{M}\left(R_{j}\llcorner a)+\mathbb{M}\left(R_{0}\llcorner a)\right.\right. \\
& \leqslant\left[\left(1-\frac{1}{s^{m}}\right)(1+\delta)+\delta\right]\left(\mathbb{M}(T\llcorner a)+2 \eta M(T))+\lambda_{0},\right.
\end{aligned}
$$

where $\lambda_{0}$ approaches 0 , as $\lambda$ tends to 0 . With a proper choice of small $\eta$ and $\delta$, these two last inequalities yield the properties b. and c. in the statement of the theorem.

3.1. Proof of the upper bound. Making use of the two results from above, we will see that the exact strategy from [ABO03, Theorem 5.6] works effectively for proving the precise lower bound of the weighted energy. Let $M$ be a current in $\mathcal{C}(\Gamma)$ and let $\delta>0$, and $s \in(1,2)$. The result is obtained by finding sequences of maps $u_{j} \in \mathrm{W}^{1, n}\left(\Omega, \mathbb{S}^{n}\right)$, closed subsets $S_{j} \subset \Omega$ of dimension $m-1$ such that $u_{j} \in \operatorname{Lip}_{\text {loc }}\left(\Omega \backslash S_{j}\right)$, and currents $R_{j} \in \mathcal{R}_{m}(\Omega)$ with the following properties:

$$
\begin{gathered}
\star \mathrm{J} u_{j}=\frac{\sigma_{n+1}}{n+1}\left(\Gamma-\partial R_{j}\right) \\
M\left(R_{j}\llcorner a) \leqslant c_{1} M\left(R_{j-1}\llcorner a), \text { with } c_{1}<1\right.\right. \\
\int_{\Omega} a(x)\left|\mathrm{D} u_{j}(x)-\mathrm{D} u_{j-1}(x)\right|^{n} \mathrm{~d} x \leqslant c_{2}^{n} M\left(R_{j-1}\llcorner a),\right.
\end{gathered}
$$

with $c_{1}$ and $c_{2}$ constants verifying $\left(\frac{c_{2}}{1-c_{1}^{1 / n}}\right)^{n}=n^{n / 2} \sigma_{n+1}+\frac{\delta}{M(M\llcorner a)}$. These sequences will be constructed by induction, starting from $u_{0}$ constant, $S_{0}=\varnothing$ and $R_{0}=M$.

Let's see first how these sequences help us prove the upper bound. From (2) and (3) we deduce that $\left(\mathrm{D} u_{j}\right)_{j}$ (modulo a subsequence) converges in $\mathrm{L}^{n}\left(\Omega, \mathbb{R}^{n+1}\right)$, so we can assume that $\left(u_{j}\right)_{j}$ converges to some map $u$ in $\mathrm{W}_{\text {loc }}^{1, n}\left(\Omega, \mathbb{S}^{n}\right)$. Hence, $\star \mathrm{J} u_{j} \rightarrow \star \mathrm{J} u$, so, by (1) and (3), we get $\star \mathrm{J} u=\frac{\sigma_{n+1}}{n+1} \Gamma$ and, by (2) and (3) and the condition on the constants $c_{1}$ and $c_{2}$, we have

$$
\int_{\Omega} a(x)|\mathrm{D} u(x)|^{n} \mathrm{~d} x \leqslant n^{n / 2} \sigma_{n+1} \mathrm{M}(M\llcorner a)+\delta .
$$

For the construction of the sequences, suppose we have $u_{j-1}, S_{j-1}$ and $R_{j-1}$ with the desired properties, for a fixed $j$. Since $S_{j-1}$ is closed, for each $x \in \Omega \backslash S_{j-1}$ we can find $r(x)>0$ such that $B_{r(x)}(x) \subset \Omega \backslash S_{j-1}$. The function $u_{j-1}$ is locally Lipschitz outside $S_{j-1}$, so we have $\left\|D u_{j-1}\right\|_{L^{\infty}\left(B_{r(x)}(x)\right)}<\infty$ and we can define

$$
0<\rho(x):=\min \left\{\frac{r(x)}{s} ; \frac{s-1}{2 s}\left\|D u_{j-1}\right\|_{L^{\infty}\left(B_{r(x)}(x)\right)}^{-1}\right\},
$$

for every $x$ in $\Omega \backslash S_{j-1}$. Then, by Theorem 2 , applied with $T=R_{j-1}$, for every small $\varepsilon>0$ we get finitely many $m$-dimensional disks $E_{r_{i}}\left(x_{i}\right)$ in $\mathbb{R}^{m+n}$ with $r_{i}<\rho\left(x_{i}\right)$ and a rectifiable $m$-current $R_{j} \subset \Omega$ such that the balls $\bar{B}_{s r_{i}}\left(x_{i}\right)$ are pairwise disjoint, and
(a) $\partial R_{j-1}=\sum_{i} \partial \llbracket E_{r_{i}}\left(x_{i}\right) \rrbracket+\partial R_{j}$
(b) $\sum_{i} \mathcal{H}^{m}\left(E_{r_{i}}\left(x_{i}\right)\right) \leqslant \frac{1+\varepsilon}{s^{m}} M\left(R_{j-1}\llcorner a)\right.$ 
(c) $\mathbb{M}\left(R_{j}\llcorner a) \leqslant\left(1-\frac{1}{s^{m+1}}\right) M\left(R_{j-1}\llcorner a)\right.\right.$.

On every $E_{r_{i}}\left(x_{i}\right)$ we insert the dipole given by Lemma 1 . More precisely, for any $\eta>0$, we take some maps $v_{i} \in \mathrm{W}^{1, n}\left(\Omega, \mathbb{S}^{n}\right)$, which are locally Lipschitz on $\Omega \backslash \partial E_{r_{i}}\left(x_{i}\right)$, constantly equal to some $y_{0} \in u_{j-1}\left(B_{s r_{i}}\left(x_{i}\right) \backslash \bar{B}_{r_{i}}\left(x_{i}\right)\right)$, and that satisfy

and thus,

$$
\begin{aligned}
\int_{\Omega} a(x)\left|\mathrm{D} v_{i}\right|^{n}(x) \mathrm{d} x & \leqslant \sigma_{n+1} n^{n / 2} \mathcal{H}_{a}^{m}\left(E_{r_{i}}\left(x_{i}\right)\right)+\eta, \\
\star \mathrm{J} v_{i} & =\frac{\sigma_{n+1}}{n+1} \partial E_{r_{i}}\left(x_{i}\right) .
\end{aligned}
$$

Because $v_{i}$ agrees with one of the values of $u_{j-1}$ on the annulus $B_{s r_{i}}\left(x_{i}\right) \backslash \bar{B}_{r_{i}}\left(x_{i}\right)$, for each $i$, we have

$$
\begin{aligned}
& \left\|u_{j-1}-v_{i}\right\|_{\mathrm{L}^{\infty}\left(B_{s r_{i}}\left(x_{i}\right) \backslash \bar{B}_{r_{i}}\left(x_{i}\right)\right)} \\
& \quad \leqslant \sup _{x, \xi \in B_{s r_{i}}\left(x_{i}\right)}\left|u_{j-1}(x)-u_{j-1}(\xi)\right|=\sup _{\substack{h \in B_{2 s r_{i}} \\
\xi \in B_{s r_{i}}\left(x_{i}\right)}}\left|u_{j-1}(\xi+h)-u_{j-1}(\xi)\right| \\
& \quad \leqslant \sup _{h \in B_{2 s r_{i}}}\left\|\mathrm{D} u_{j-1}\right\|_{\mathrm{L}^{\infty}\left(B_{s r_{i}}\left(x_{i}\right)\right)}|h|=2 s r_{i}\left\|\mathrm{D} u_{j-1}\right\|_{\mathrm{L}^{\infty}\left(B_{s r_{i}}\left(x_{i}\right)\right) .}
\end{aligned}
$$

By the choice of $\rho$, this implies $\left|u_{j-1}-v_{i}\right| \leqslant s-1 \leqslant 1$ a.e. on $B_{s r_{i}}\left(x_{i}\right) \backslash \bar{B}_{r_{i}}\left(x_{i}\right)$, and the balls $B_{s r_{i}}\left(x_{i}\right)$ being pairwise disjoint, we can repeatedly apply Lemma 5.4 in [ABO03] to get the maps $w_{i} \in \mathrm{W}^{1, n}\left(\Omega, \mathbb{S}^{n}\right)$ with

$$
w_{i}=\left\{\begin{array}{ll}
v_{i} & \text { on } B_{r_{i}}\left(x_{i}\right) \\
w_{i-1} & \text { on } \Omega \backslash B_{s r_{i}}\left(x_{i}\right)
\end{array}, \quad \text { where } w_{0} \equiv u_{j-1} .\right.
$$

Since there are a finite number of balls $B_{r_{i}}\left(x_{i}\right)$, we can take $u_{j}$ to be the last $w_{i}$, that is,

$$
u_{j}= \begin{cases}v_{i} & \text { on } B_{r_{i}}\left(x_{i}\right), \forall i \\ u_{j-1} & \text { on } \Omega \backslash \bigcup_{i} B_{s r_{i}}\left(x_{i}\right)\end{cases}
$$

moreover, Lemma 5.4 in [ABO03] also gives us $\mathrm{J} u_{j}=\mathrm{J} u_{j-1}+\sum_{i} \mathrm{~J} v_{i}$, and

$$
\left\|\mathrm{D} u_{j}\right\|_{\mathrm{L}^{\infty}\left(B_{s r_{i}}\left(x_{i}\right) \backslash \bar{B}_{r_{i}}\left(x_{i}\right)\right)} \leqslant \frac{2}{\sqrt{3}} \frac{3 s-1}{s-1}\left\|\mathrm{D} u_{j-1}\right\|_{\mathrm{L}^{\infty}\left(B_{s r_{i}}\left(x_{i}\right)\right)}, \forall i
$$

By construction (see the proof of Lemma 5.4 in [ABO03]), the function $u_{j}$ is locally Lipschitz outside $S_{j}:=\bigcup_{i} \partial E_{r_{i}}\left(x_{i}\right) \cup S_{j-1}$. Properties (a) and (c) show that $u_{j}$ satisfies the required conditions (30), and (31) with $c_{1}=1-\frac{1}{s^{m+1}}$, respectively, and it remains to check (32). We 
have the estimate

$$
\begin{aligned}
& \left(\int_{\Omega} a(x)\left|\mathrm{D} u_{j}(x)-\mathrm{D} u_{j-1}(x)\right|^{n} \mathrm{~d} x\right)^{1 / n} \\
& \leqslant\left(\sum_{i} \int_{B_{s r_{i}}\left(x_{i}\right) \backslash \bar{B}_{r_{i}}\left(x_{i}\right)} a\left|\mathrm{D} u_{j}\right|^{n}+\sum_{i} \int_{B_{r_{i}}\left(x_{i}\right)} a\left|\mathrm{D} v_{i}\right|^{n}\right)^{1 / n} \\
& +\left(\sum_{i} \int_{B_{s r_{i}}\left(x_{i}\right)} a\left|\mathrm{D} u_{j-1}\right|^{n}\right)^{1 / n} \\
& \leqslant\left[\left(\frac{3 s-1}{\sqrt{3} s}\right)^{n} \sum_{i} \frac{1}{r_{i}^{n}} \int_{B_{s r_{i}}\left(x_{i}\right) \backslash \bar{B}_{r_{i}}\left(x_{i}\right)} a(x) \mathrm{d} x\right. \\
& \left.+n^{n / 2} \sigma_{n+1} \sum_{i} \mathcal{H}_{a}^{m}\left(E_{r_{i}}\left(x_{i}\right)\right)+\sum_{i} \eta\right]^{1 / n}+\frac{s-1}{2 s}\left(\sum_{i} \frac{1}{r_{i}^{n}} \int_{B_{r_{i}}\left(x_{i}\right)} a(x) \mathrm{d} x\right)^{1 / n}
\end{aligned}
$$

Taking into account property (b), it is clear that there exist $\varepsilon>0, \eta>0$ and $s \in(1,2)$, such that condition (32) is verified, with the constant $c_{2}$ also satisfying the required inequality relative to $c_{1}$.

3.2. Proof of the lower bound. As we mentioned in the introduction, the lower bound can be obtained with the help of the coarea formula, like in [ABO03], and, before that, as seen in [ABL88]. Let $u: \Omega \rightarrow \mathbb{S}^{n}$ be an element of $\mathcal{E}(\Gamma)$, that is, $u$ belongs to $\mathrm{W}^{1, n}\left(\Omega, \mathbb{S}^{n}\right)$ and it satisfies $\star \mathrm{J} u=\frac{\sigma_{n+1}}{n+1} \Gamma$. We can choose, recalling (19), a point $z \in \mathbb{S}^{n}$ such that $\star \mathrm{J} u=\frac{\sigma_{n+1}}{n+1} \llbracket N_{z} \rrbracket$, and which verifies the inequality

$$
\int_{N_{z}} a(x) \mathrm{d} \mathcal{H}^{m}(x) \leqslant \frac{1}{\sigma_{n+1}} \int_{\mathbb{S}^{n}}\left(\int_{N_{y}} a(x) \mathrm{d} \mathcal{H}^{m}(x)\right) \mathrm{d} \mathcal{H}^{n}(y) .
$$

By the coarea formula (15), and inequality (10), we have

$$
\begin{aligned}
\int_{\mathbb{S}^{n}}\left(\int_{N_{y}} a(x) \mathrm{d} \mathcal{H}^{m}(x)\right) \mathrm{d} \mathcal{H}^{n}(y) & =\int_{\Omega} a(x)\left|u^{\sharp} \omega_{0}(x)\right| \mathrm{d} x \\
& \leqslant n^{-n / 2} \int_{\Omega} a(x)|\mathrm{D} u(x)|^{n} \mathrm{~d} x .
\end{aligned}
$$

Since the current $M_{0}:=(-1)^{m} \llbracket N_{z} \rrbracket$ belongs to the class $\mathcal{C}(\Gamma)$, we have

$$
\int_{N_{z}} a(x) \mathrm{d} \mathcal{H}^{m}(x)=\mathbb{M}\left(M_{0}\llcorner a)=\mathbb{M}\left(\llbracket N_{z}\llcorner a \rrbracket) \geqslant \inf _{M \in \mathcal{C}(\Gamma)} \mathbb{M}(M\llcorner a),\right.\right.
$$

and therefore we have obtained

$$
\int_{\Omega} a(x)|\mathrm{D} u(x)|^{n} \mathrm{~d} x \geqslant n^{n / 2} \sigma_{n+1} \inf _{M \in \mathcal{C}(\Gamma)} M(M\llcorner a),
$$

which completes the proof of the theorem.

Acknowledgements. I am indebted to Prof. Petru Mironescu, for proposing the problem and for productive discussions and suggestions. I am grateful to Pierre Bousquet for being open for discussions during a summer school in Marseille in 2011, for helping me prove one of the results on distributional Jacobian, and for its critical reading of the manuscript. I also thank Vincent Borrelli for the differential geometry lessons. 


\section{REFERENCES}

[AAR99] George E. Andrews, Richard Askey, and Ranjan Roy. Special functions, volume 71 of Encyclopedia of Mathematics and its Applications. Cambridge University Press, Cambridge, 1999.

[ABL88] F. Almgren, W. Browder, and E. H. Lieb. Co-area, liquid crystals, and minimal surfaces. In Partial differential equations (Tianjin, 1986), volume 1306 of Lecture Notes in Math., pages 1-22. Springer, Berlin, 1988.

[ABO03] G. Alberti, S. Baldo, and G. Orlandi. Functions with prescribed singularities. J. Eur. Math. Soc. (JEMS), 5(3):275-311, 2003.

[AFP00] Luigi Ambrosio, Nicola Fusco, and Diego Pallara. Functions of bounded variation and free discontinuity problems. Oxford Mathematical Monographs. The Clarendon Press Oxford University Press, New York, 2000.

[Alb05] Giovanni Alberti. Distributional Jacobian and singularities of Sobolev maps. Ricerche Mat., 54(2):375-394 (2006), 2005.

[BC83] Haïm Brezis and Jean-Michel Coron. Large solutions for harmonic maps in two dimensions. Comm. Math. Phys., 92(2):203-215, 1983.

[BCL86] Haïm Brezis, Jean-Michel Coron, and Elliott H. Lieb. Harmonic maps with defects. Comm. Math. Phys., 107(4):649-705, 1986.

[Bet90] F. Bethuel. A characterization of maps in $\mathrm{H}^{1}\left(\mathbb{B}^{3}, \mathbb{S}^{2}\right)$ which can be approximated by smooth maps. Ann. Inst. H. Poincaré Anal. Non Linéaire, 7(4):269-286, 1990.

[Bou07] Pierre Bousquet. Topological singularities in $\mathrm{W}^{s, p}\left(\mathbb{S}^{N}, \mathbb{S}^{1}\right)$. J. Anal. Math., 102:311-346, 2007.

[EG92] Lawrence C. Evans and Ronald F. Gariepy. Measure theory and fine properties of functions. Studies in Advanced Mathematics. CRC Press, Boca Raton, FL, 1992.

[Fed59] Herbert Federer. Curvature measures. Trans. Amer. Math. Soc., 93:418-491, 1959.

[Fed69] Herbert Federer. Geometric measure theory. Die Grundlehren der mathematischen Wissenschaften, Band 153. Springer-Verlag New York Inc., New York, 1969.

[GMS98] Mariano Giaquinta, Giuseppe Modica, and Jiří Souček. Cartesian currents in the calculus of variations. I, volume 37 of Ergebnisse der Mathematik und ihrer Grenzgebiete. 3. Folge. A Series of Modern Surveys in Mathematics [Results in Mathematics and Related Areas. 3rd Series. A Series of Modern Surveys in Mathematics]. Springer-Verlag, Berlin, 1998. Cartesian currents.

[JS02] R. L. Jerrard and H. M. Soner. Functions of bounded higher variation. Indiana Univ. Math. J., 51(3):645-677, 2002.

[Laf96] J. Lafontaine. Introduction aux variétés différentielles. Collection Grenoble sciences. EDP Sciences, 1996.

[Mil05] Vincent Millot. Energy with weight for $\mathbb{S}^{2}$-valued maps with prescribed singularities. Calc. Var. Partial Differential Equations, 24(1):83-109, 2005.

[Mor09] Frank Morgan. Geometric measure theory. Elsevier/Academic Press, Amsterdam, fourth edition, 2009. A beginner's guide.

[Pak01] Mohammad Reza Pakzad. Existence of infinitely many weakly harmonic maps from a domain in $\mathbb{R}^{n}$ into $\mathbb{S}^{2}$ for non-constant boundary data. Calculus of Variations and Partial Differential Equations, 13:97-121, 2001

[Pap02] E. Pap, editor. Handbook of measure theory. Vol. I, II. North-Holland, Amsterdam, 2002. 